\title{
Extravascular CD3+ T Cells in Brains of Alzheimer Disease Patients Correlate with Tau but Not with Amyloid Pathology: An Immunohistochemical Study
}

\author{
Mario Merlini Tunahan Kirabali Luka Kulic Roger M. Nitsch \\ Maria Teresa Ferretti \\ Institute for Regenerative Medicine (IREM), University of Zurich, Switzerland Neuroscience Center Zurich (ZNZ), \\ Zurich, Switzerland
}

\section{Keywords}

Alzheimer disease - T cells · CD3 - Amyloid - Tau - AT8 - 6E10 . Hippocampus · Mid frontal gyrus

\begin{abstract}
Background: Strong genetic and epidemiological evidence points to a crucial role of the immune system in the development of Alzheimer disease (AD). CD3+ T lymphocytes have been described in brains of postmortem AD patients and in transgenic models of AD-like cerebral amyloidosis and tau pathology. However, the occurrence of T cells in AD brains is still controversial; furthermore, the relationship between $T$ cells and hallmarks of AD pathology (amyloid plaques and neurofibrillary tangles) remains to be established. Objectives: We have studied the occurrence of T cells in postmortem hippocampi and mid frontal gyrus (MFG) samples of AD patients (Braak stage $\mathrm{V}-\mathrm{VI}$ ) and nondemented control subjects and correlated it with amyloid and tau pathology burden. Methods: Confocal microscopy and bright-field immunohistochemistry were used to identify brain-associated T cells. Extravascular CD3+ T cells were quantified and compared to nondemented controls. In ad-
\end{abstract}

\section{KARGER}

(C) 2018 S. Karger AG, Basel

E-Mail karger@karger.com

www.karger.com/ndd dition, numbers of extravascular CD3+ T cells were correlated with amyloid (6E10 staining) and tau pathology (AT8 staining) in the same sections. Results: Several CD3+, extravascular T cells were observed in the brains of AD patients, mostly of the CD8+ subtype. AD hippocampi harbored significantly increased numbers of extravascular CD3+ T cells compared to nondemented controls. CD3+ T cells significantly correlated with tau pathology but not with amyloid plaques in AD samples. Conclusions: Our data support the notion of T-cell occurrence in AD brains and suggest that, in advanced stages of $A D, T$-cell extravasation is driven by taurelated neurodegenerative changes rather than by cerebral amyloidosis. T cells could be crucial for driving the amyloidindependent phase of the AD pathology.

(c) 2018 S. Karger AG, Basel

\section{Introduction}

Alzheimer disease (AD), the leading cause of dementia in the elderly, is a complex, multifactorial disease with a strong inflammatory component [1]. While the role of microglial cells (the resident immune cells of the brain) 
Table 1. Patient characteristics

\begin{tabular}{lll}
\hline & $\begin{array}{l}\text { Nondemented } \\
\text { controls }\end{array}$ & $\begin{array}{l}\text { Alzheimer } \\
\text { disease } \\
\text { patients }\end{array}$ \\
\hline $\begin{array}{l}\text { Subjects, } n \text { (\% females) } \\
\text { Age, years }\end{array}$ & $10(60)$ & $9(100)$ \\
APOE status & $84 \pm 1.9$ & $84.1 \pm 3.6$ \\
$3 / 2$ & $1(10)$ & 0 \\
$3 / 3$ & $6(60)$ & $3(33.3)$ \\
$3 / 4$ & $2(20)$ & $4(44.4)$ \\
$4 / 4$ & 0 & $2(22.2)$ \\
u.d. & $1(10)$ & 0 \\
CERAD & $2(20)$ & 0 \\
0 & $6(60)$ & 0 \\
A & $2(20)$ & 0 \\
B & 0 & $9(100)$ \\
C & & 0 \\
Braak stage & $7(70)$ & 0 \\
1 & $2(20)$ & 0 \\
2 & $1(10)$ & 0 \\
3 & 0 & $3(33.3)$ \\
4 & 0 & $6(66.7)$ \\
5 & 0 & \\
6 & & \\
\hline
\end{tabular}

Values are presented as numbers (\%) or means \pm SD unless otherwise stated. CERAD, Consortium to Establish a Registry for Alzheimer's Disease; u.d., undetermined.

has been actively studied, very little is known about the contribution of additional immune cells, such as T lymphocytes, to AD pathology $[2,3]$.

In fact, in contrast with the original concept of being an immune-privileged organ, it is becoming increasingly clear that the brain is under tight control by the peripheral adaptive immune system [4], which is crucially involved in maintaining adult neurogenesis $[5,6]$ as well as mounting immune reactions during challenge [7]. Indeed, while they are normally absent from the brains of healthy, young individuals, parenchymal CD3+ T cells have been consistently described in postmortem brains of $\mathrm{AD}$ patients [8-11].

The occurrence and significance of T cells in AD brains is still controversial; furthermore, the relationship between T cells and hallmarks of AD (extracellular deposits of $\beta$-amyloid peptide $(A \beta)$ and intracellular aggregates of abnormally phosphorylated tau) remains to be established. Here, we have reassessed the occurrence of T cells in postmortem $\mathrm{AD}$ brains and correlated it with markers of tau and amyloid pathology.

\section{Materials and Methods}

\section{Cases}

Paraffin-embedded hippocampal tissue blocks of 10 nondemented and $9 \mathrm{AD}$ cases (Braak stage $\mathrm{V}-\mathrm{VI}$ ) were obtained from the Netherlands Brain Bank (NBB), Netherlands Institute for Neuroscience, Amsterdam, The Netherlands. For some of the individuals, mid frontal gyrus (MFG) samples were also available (AD, $n=5$; non-demented, $n=8)$. All material was collected from donors for or from whom a written informed consent for a brain autopsy and the use of the material and clinical information for research purposes had been obtained by the NBB. The subjects' clinical characteristics are presented in Table 1.

\section{Immunohistochemistry}

Confocal Microscopy. Following standard deparaffinization and rehydration steps, hippocampal AD brain paraffin sections (20- $\mu \mathrm{m}$ thick) were pretreated with citrate antigen retrieval buffer for $20 \mathrm{~min}$ at $95^{\circ} \mathrm{C}$ as previously published [12]. Subsequently, the sections were immune-blocked and incubated with either biotinylated GSL I-isolection B4 diluted 1:500 (B-1205; Vector Laboratories/Reactolab SA, Servion, Switzerland) and rabbit anti-human CD3 antibody diluted 1:200 (A0452; DAKO Switzerland, Baar, Switzerland) or biotinylated GSL I-isolection B4 and rabbit antihuman CD8 antibody diluted 1:400 (108R-16; Cell Marque, Rocklin, CA, USA) for $2 \mathrm{~h}$ at RT. The sections were subsequently incubated with Cy2-conjugated streptavidin (diluted 1:800; Jackson ImmunoResearch/Milan Analytica AG, Rheinfelden, Switzerland) and Cy3-conjugated donkey anti-rabbit secondary antibody (diluted 1:700; Jackson ImmunoResearch/Milan Analytica AG) for $1 \mathrm{~h}$ at room temperature, followed by incubation with DAPI diluted 1:5,000 for 5 min at RT and 0.2\% Sudan Black in 50\% ethanol for $10 \mathrm{~min}$ at room temperature. The sections were coverslipped using Hydromount ${ }^{\circledR}$ mounting medium (National Diagnostics, Atlanta, GA, USA).

$\mathrm{Z}$-stack images of the stained sections were acquired at an emission and excitation wavelength of 405,488 , and $561 \mathrm{~nm}$ and 460 , 520 , and $570 \mathrm{~nm}$ for DAPI, Cy2, and Cy3, respectively, using confocal microscopy (Leica SP8; Leica, Wetzlar, Germany) in sequential imaging mode. The acquired images were converted into maximum $\mathrm{Z}$-stack projection images and $3-\mathrm{D}$ volume renderings of the maximum z-stack projections in ImageJ (National Institutes of Health, Bethesda, MD, USA).

Double Bright-Field Immunohistochemistry and 6E10 Staining of Plaques. Sections ( $5 \mu \mathrm{m}$ thick) were deparaffinized and rehydrated. Following antigen retrieval with citrate buffer and proteinase $\mathrm{K}$, the sections were blocked in $10 \%$ donkey serum in TBS-T for $1 \mathrm{~h}$ at room temperature and incubated with rabbit anti-human CD3 antibody (A0452; DAKO, Glostrup, Denmark) for $2 \mathrm{~h}$ at RT. Afterwards they were incubated with secondary biotinylated donkey anti-rabbit antibody and further processed using the VectaStain ${ }^{\circledR}$ Elite staining and nickel-enhanced DAB substrate. The sections were subsequently incubated with mouse anti-human collagen IV antibody (M0785; DAKO, Glostrup, Denmark) for $2 \mathrm{~h}$ at $\mathrm{RT}$ and processed as described for the CD3 staining, with the use of nonenhanced DAB substrate. Hippocampal and MFG sections adjacent to the ones stained for CD3 and collagen IV were processed and incubated with rabbit anti-human CD3 and mouse anti-human phospho-tau antibody (AT8, diluted 1:200; Fisher Scientific, Wohlen, Switzerland) as described above using nickel-en- 
hanced and nonenhanced $\mathrm{DAB}$ as a substrate for the tau and CD3 staining, respectively. Another set of adjacent sections was incubated with mouse anti-A $\beta$ 6E10 antibody (diluted 1:500; LucernaChem, Luzern, Switzerland) as described above. Microscopy images were acquired from the A $\beta$-, tau-, CD3-, and collagen IVstained sections using a Leica DM4000B microscope (Leica) equipped with an Olympus DP71 digital camera (Olympus, Volketswil, Switzerland) in bright-field (tau-, CD3-, and collagen IV-stained sections) or fluorescent (A $\beta$-stained sections) mode.

\section{Quantification}

The number of $A \beta$ plaques, paired helical filament tau-positive structures (including neurofibrillary tangles, tau-positive perycaria and neuritic plaques, as shown in Fig. 2A), and intra- and extravascular $\mathrm{CD} 3+\mathrm{T}$ cells was quantified with a $\times 20$ objective using the newCAST module of Visiopharm software (Visiopharm, Hørsholm, Denmark). A region of interest was drawn around the entire section and the automatic stage was set to randomly move within the region of interest, covering $50 \%$ of the section's area. CD3- and tau-positive cells and $\mathrm{A} \beta$ plaques were counted by the experimenter, blind to the origin of the samples and normalized to the size of the respective brain section.

\section{Statistical Analysis}

Two-way ANOVA was used to study the effect of brain region and diagnosis on numbers of $\mathrm{CD} 3+\mathrm{T}$ cells. Bonferroni post hoc multiple comparisons were used to determine statistically significant differences. Correlations were analyzed with Pearson's correlation test and coefficients of determination $\left(R^{2}\right)$ of linear regression were calculated (all analyses were performed with Prism version 5.0)

\section{Online Supplementary Methods}

See www.karger.com/doi/10.1159/000486200 for all online suppl. material.

CD3/CD8 Quantification Study. For this study we used 5- $\mu \mathrm{m}-$ thick hippocampal sections from 9 subjects (4 nondemented controls and $5 \mathrm{AD}$ patients). The sections ( 2 per subject) were processed as indicated above for confocal immunofluorescence staining. The primary antibodies used were: rabbit anti-CD3 (A0452; DAKO, Glostrup, Denmark; working dilution 1:200), mouse antihuman CD8 alpha (ab17147; Abcam, USA; working dilution 1:50), and donkey anti-mouse Cy3 and anti-rabbit Alexa488 (both from Jackson Laboratories, USA; working dilution 1:200). For quantification, 10 microscopy images ( 5 for CD8 and 5 for CD3) were acquired from each section using a Leica DM4000B microscope (Leica) equipped with an Olympus DP71 digital camera (Olympus, Volketswil, Switzerland). CD3+ and CD8+ cells were manually counted in each image; the numbers were added up to generate the total number of CD3+ and CD8+ cells per subject per slide. Data are shown as the percentage of CD8+ cells over the total number of CD3+ cells.

Granzyme B and Caspase 3 Study. The staining protocol was optimized on human, formalin-fixed, and paraffin-embedded tonsil material, which was obtained anonymized from the tissue biobank of the Institute of Pathology and Molecular Pathology, University Hospital Zürich. The primary antibodies used were: mouse anti-human granzyme B (clone GBZ01; Thermo Fisher Scientific, USA; working dilution 1:50) and rabbit anti-cleaved caspase 3 (Cell Signaling, USA; working dilution 1:300).

T Cells and Tau in Alzheimer Brains

\section{Results}

Increased Occurrence of T Cells in Postmortem AD Brain Parenchyma

To reassess the occurrence of $\mathrm{T}$ cells in $\mathrm{AD}$ brains, we first used hippocampal samples from advanced AD stage subjects (Braak stages V and VI), as these have been described to harbor the highest number of cerebral $\mathrm{T}$ cells [10]. Triple immunostainings were used to identify CD3+ or CD8+ T cells with respect to endothelial cells (stained with isolectin 4) or astrocytes (GFAP).

Several extravascular CD3+ T cells were observed in the samples (Fig. 1A-C); on average, $90 \%$ of all of the CD3+ $\mathrm{T}$ cells observed were CD8+ (see online suppl. Fig. 1A, B). The morphology of CD3+ T cells varied greatly; most cells showed a typical small and round-shaped morphology, but we also observed cell protrusions consistent with uropod formation and large cells with an intense cytoplasmic CD3 signal (Fig. 1B). Even though such morphologies could suggest activation of infiltrated $\mathrm{T}$ cells, no granzyme B (typical marker of effector CD8+ T cells) was detectable in brain AD tissue, while it was readily observed in human tonsils (see online suppl. Fig. 1C and data not shown). Furthermore, cleaved caspase 3 was not observed in AD brain samples, in contrast to the clear staining obtained in human tonsil samples (see online suppl. Fig. 1C and data not shown).

Next, the numbers of CD3 + T cells were quantified in paired hippocampal and MFG postmortem brain samples from advanced $\mathrm{AD}$ (Braak stage V-VI) subjects and nondemented age-matched controls. We used double bright-field immunohistochemistry to detect $\mathrm{T}$ cells $(\mathrm{CD} 3+)$ and vessels (stained with collagen IV). Although extravascular CD3 + T cells were detected in all samples (Fig. 1C), their numbers were significantly increased in $\mathrm{AD}$ brains compared with those in controls (Fig. 1C, 2 -way ANOVA, $p<0.05$ ), and specifically in hippocampi (Bonferroni's post hoc test, $p<0.01$ ), as previously shown [10]. Increased numbers of infiltrated CD3+ T cells were observed in hippocampi of $\mathrm{AD}$ cases as compared to nondemented controls, regardless of the ApoE genotype (see online suppl. Fig. 1D). The number of T cells in MFG was not statistically different between AD and controls.

\section{Correlation with Pathology}

To explore the relationship between T-cell invasion and AD pathology, we assessed the amyloid burden (as indicated by the number of $6 \mathrm{E} 10+$ plaques) and pairedhelical filament tau (measured by AT8+ immunoreactive

Neurodegener Dis 2018;18:49-56 DOI: $10.1159 / 000486200$ 


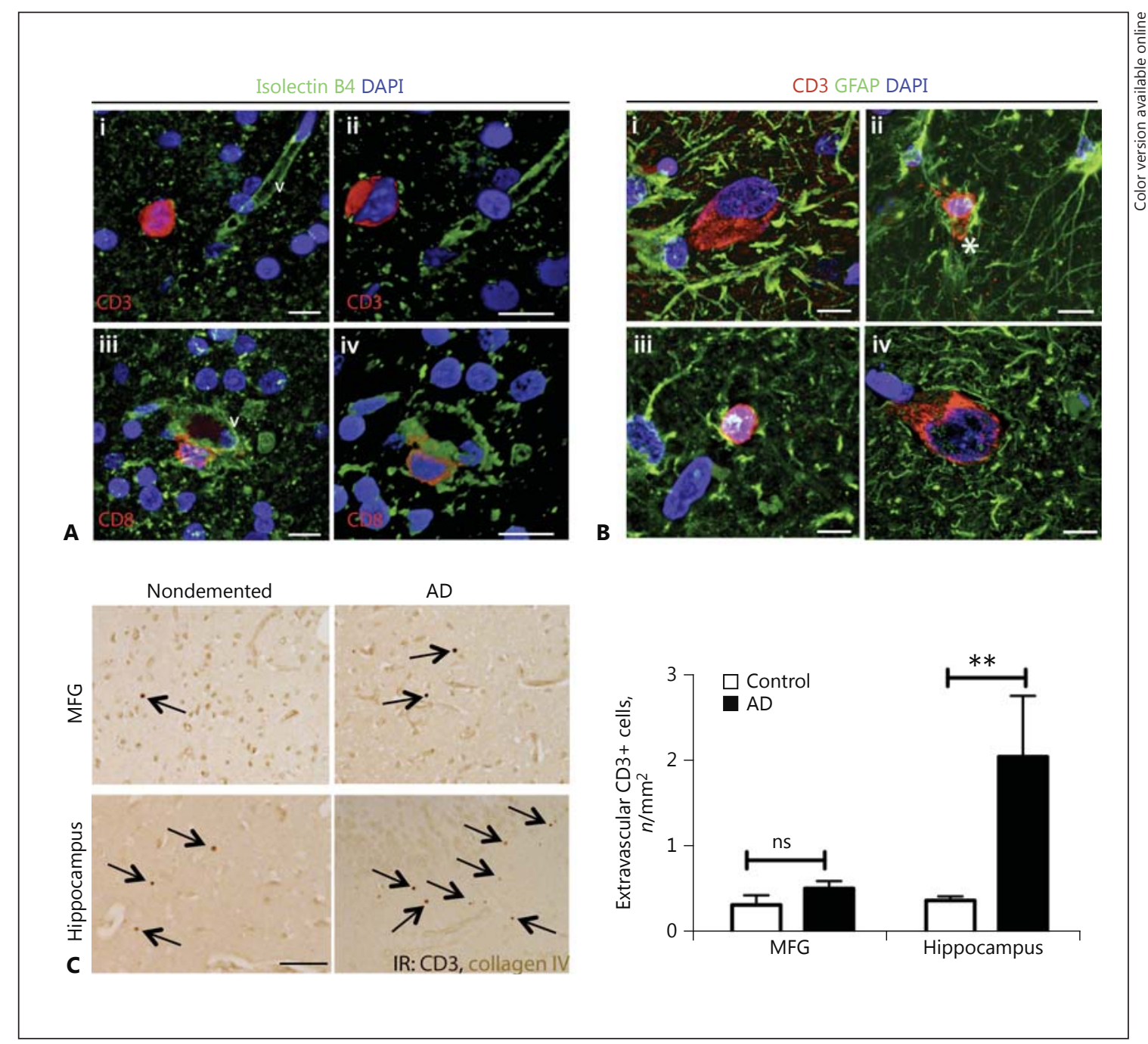

Fig. 1. Occurrence of CD3+ extravascular T cells in Alzheimer disease (AD) brains. A Representative confocal microscopy maximum $\mathrm{z}$-stack projection images showing hippocampus-infiltrated CD3+ (i, ii) and CD8+ (iii, iv) T cells in $20-\mu \mathrm{m}$-thick sections from a Braak stage VI Alzheimer disease subject. T cells were located outside the lumen of vessels ( $\mathrm{v}$, revealed with isolectin B4 staining), confirming their infiltration into brain parenchyma. ii and iv are a 3 -D volume rendering of the maximum $\mathrm{z}$-stack projection shown in $\mathbf{i}$ and iii, respectively, identifying the presence of a nucleus within the structure stained by the anti-CD3 and CD8 antibodies. Scale bars, $10 \mu \mathrm{m}$. B Representative confocal microscopy maximum $\mathrm{z}$-stack projection images illustrating the heterogeneity of $\mathrm{T}$ cells detected in AD brains. Astrocytes (GFAP staining) are shown for comparison. Together with small, round, resting $\mathrm{T}$ cells with

structures, including neuritic plaques, perikarya, and tangles) in each sample (Fig. 2A). As expected [13] tau pathology was very rare in nondemented cases, with the hippocampus more affected than the MFG. In contrast, AD typical membrane-associated CD3 staining (iii), we also observed cell protrusions consistent with uropod formation (ii, asterisk) and large cells with an intense cytoplasmic CD3 signal (i, iv) indicative of activation. Scale bars, $10 \mu \mathrm{m}$. C Representative micrographs illustrating T-cell occurrence (CD3, dark brown stain, arrows) in relation to vessels (collagen IV, light brown) in 5- $\mu$ m-thick sections of hippocampi and mid frontal gyrus (MFG) from AD patients and nondemented controls. Scale bar, $200 \mu \mathrm{m}$. CD3+ T cells were found to be upregulated in AD hippocampi $\left({ }^{* *} p<0.01\right.$, 2-way ANOVA with Bonferroni's post hoc test; $n=9, \mathrm{AD} ; n=10$, nondemented controls), but not MFG $(n=5, \mathrm{AD} ; n=8$, nondemented controls), compared to nondemented controls. ns, not significant.

cases and in particular AD hippocampi had a very severe tau pathology. Two-way ANOVA confirmed that the tau pathology burden was significantly affected by diagnosis and brain region (effect of diagnosis and brain region: 
$p<0.001$; diagnosis $\times$ brain region interaction: $p<0.001$; Fig. 2B). Hippocampi displayed significantly more tau pathology than MFG samples, an effect that was highly significant in $\mathrm{AD}$ cases $(p<0.001$, Bonferroni's post hoc test; Fig. 2B) and is in line with the typical temporal progression of tau pathology in neurodegeneration [14].

In contrast, several amyloid plaques were observed in hippocampi and MFG of both AD and nondemented cases (Fig. 2A). Even though MFG from AD cases tended to have a higher amyloidosis than nondemented controls, a 2-way ANOVA did not identify any statistical difference in amyloid burden due to brain region or diagnosis (Fig. 2B).

When we studied the correlation between the number of extravascular CD3+ T cells in each sample and either amyloid or tau pathology, we found that, overall, T cells significantly correlated with tau pathology (Fig. 2C; $p<$ 0.001 , Pearson's correlation, $R^{2}=0.56$ ) but not with the number of $A \beta$ plaques. We further explored the contribution of diagnosis and brain region to this correlation. We found that CD3+ T cells significantly correlated with tau, but not with amyloid, in AD samples analyzed separately; here, the correlation was mostly driven by the hippocampal samples $\left(p<0.001\right.$, Pearson's correlation, $\left.R^{2}=0.55\right)$. In contrast, in nondemented cases analyzed as a separate group, we observed an overall weak correlation with amyloid pathology ( $p<0.05$, Pearson's correlation, $R^{2}=$ 0.22 ) while no effect of tau was detected. Even though a similar trend was observed in both the hippocampi and the MFG of nondemented patients, none of the correlations reached significance when the brain areas were analyzed individually.

Interestingly, no physical association was observed between $\mathrm{T}$ cells and tau costained on the same section.

\section{Discussion}

Our findings add to a consistent body of evidence in the literature, indicating increased T cells, mostly CD8+ lymphocytes, in brain parenchyma of AD [8-10]. In addition, several studies have indicated alterations in both peripheral and CNS-associated T cells in $\mathrm{AD}$ patients compared to controls. An increased A $\beta$-specific CD4-Tcell response was found in blood-derived samples from $\mathrm{AD}$ patients, suggesting that $\mathrm{T}$ cells can sense the $\mathrm{AD}$ pathology [15]. Two recent studies identified a proinflammatory, T-cell-mediated profile in CSF of AD patients [16] and of amnestic MCI [17], a condition considered prodromal to $\mathrm{AD}$. In addition, white-matter-as-

T Cells and Tau in Alzheimer Brains sociated T cells (largely of the CD8+ type) have been observed via immunohistochemistry and flow-cytometry in white matter from $\mathrm{AD}$ and other neurological diseases [18].

T-cell activation in the periphery and infiltration into the CSF and brain could therefore be an important player in the $\mathrm{AD}$ pathology. In spite of their low numbers, parenchymal $\mathrm{T}$ cells might be able to significantly impact the CNS milieu by releasing pro- or anti-inflammatory cytokines; since the vast majority of $\mathrm{CD} 3+\mathrm{T}$ cells were CD8+ (see online suppl. Fig. 1A, B), direct cytotoxic activity might take place. Even though we observed morphological signs of activation (Fig. 1B), we did not detect expression of granzyme B in the brain (see online suppl. Fig. 1C and data not shown), indicating a low overall effector function of CD8+ T cells. While the exact phenotype of infiltrated $\mathrm{T}$ cells in $\mathrm{AD}$ brains remains to be carefully characterized, based on our data we conclude that infiltrated CD8+ T cells are not inducing granzyme-Bmediated apoptosis of target cells; this view is supported by the lack of cleaved caspase- 3 expression in our sample set (see online suppl. Fig. 1C and data not shown). We speculate that initial activation of $\mathrm{T}$ cells is needed for their extravasation and infiltration into the brain parenchyma; once in the tissue, the cells likely downregulate activation markers. Previous studies in human $[10,18]$ and animal models [19] have indeed revealed an inhibited phenotype of brain-infiltrated $\mathrm{T}$ cells.

It is still unclear what drives T-cell cerebral infiltration in AD. Misfolded amyloid and tau can independently lead to T-cell extravasation as shown in transgenic animal models of cerebral amyloidosis [19] and tau pathology [20]. However, in line with previous reports $[10,19,20]$ we observed no physical association between $\mathrm{T}$ cells and either amyloid deposits or tau lesions, suggesting that $\mathrm{T}$ cells are not specifically attracted to them. It is rather likely that T-cell infiltration is a downstream event of $\mathrm{AD}$ neuropathology, following microglial and endothelial activation and vascular remodeling. Since both amyloid [21] and tau [22] can induce microglial activation, microglial release of chemokines such as CCL5 (RANTES), CCL4 (MIP-1 $\beta$ ), and CCL2 (MCP-1) might drive T-cell recruitment to $\mathrm{AD}$ brains (as proposed in multiple sclerosis [23]). Inflammatory chemokines might also be expressed by activated endothelial cells; indeed endothelial activation has been shown to occur in $\mathrm{AD}$ [24] and to accompany amyloid pathology and T-cell infiltration in the context of cerebral amyloidosis in animal models of ADlike amyloidosis [19]. Finally, tau pathology has been recently shown to affect vessel wall remodeling of lepto-

Neurodegener Dis 2018;18:49-56 DOI: $10.1159 / 000486200$ 


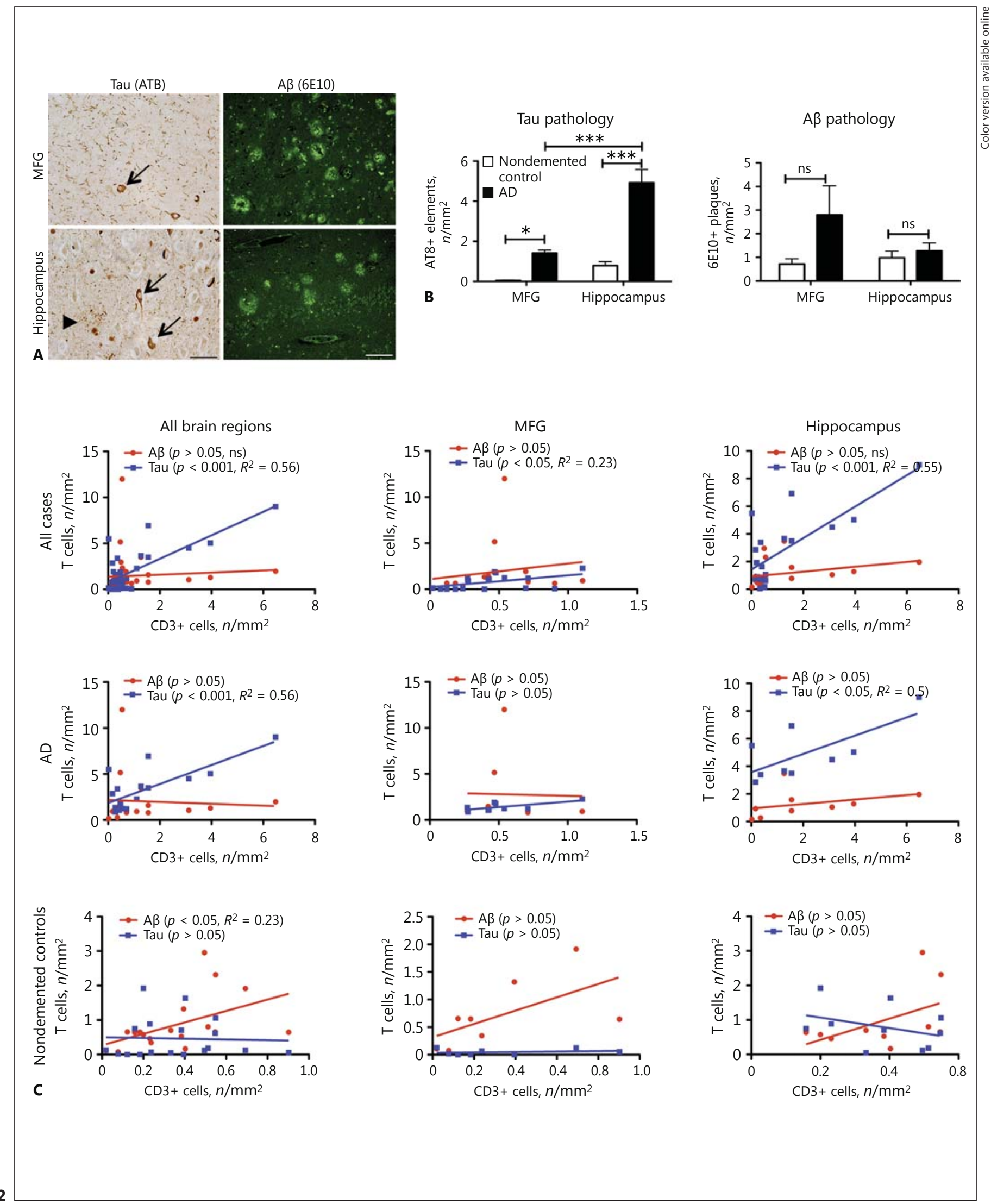

(For legend see next page.) 
meningeal arteries [25]; tau-induced arterial smooth muscle and elastin loss might underlie vascular frailty, microvessel instability, and increased permeability at the pre- and postcapillary level. In addition, the ApoE allelic state might modulate T-cell infiltration. Even though our sample set did not allow for a proper statistical analysis of data stratified by ApoE genotype, it appeared that ApoEc4 homozygous $\mathrm{AD}$ cases harbored the highest number of infiltrated T cells as compared to the other genotypes (see online suppl. Fig. 1D). The effect of ApoEs4 on T-cell numbers might depend on the interaction with cardiovascular comorbidities (which can weaken the bloodbrain barrier and are associated with $\mathrm{AD}$ diagnosis in ApoEe 4 carriers [26]) or on exacerbated tau pathology, as indicated in recent studies [27].

Our analysis allowed us to collect correlative evidence of the relationship between $\mathrm{CD} 3+\mathrm{T}$ cells and $\mathrm{AD}$ lesions in postmortem human brain samples. Since CD8+ cells represented $90 \%$ of $\mathrm{CD} 3+\mathrm{T}$ cells detected in brains, the correlations generated for $\mathrm{CD} 3+\mathrm{T}$ cells are largely representative of the CD8+ T cell population. However, detailed quantifications of each cellular subset (CD4+ and CD8+) with both tau and amyloid pathology would be of interest and should be addressed in future studies.

Overall, we found that the number of extravascular CD3 + T cells correlated with tau, but not with amyloid pathology. However, we found that this correlation highly depended on the diagnosis and the brain region analyzed (Fig. 2C). Indeed, the correlation was very strong in $\mathrm{AD}$ cases, and specifically in hippocampi. The correlation with tau likely explained why $\mathrm{T}$ cell extravasation was more pronounced in $\mathrm{AD}$ hippocampi, harboring a more advanced tau pathology than the MFG (Fig. 2A, B and Togo et al. [10]).

Our data are in line with those of Zotova et al. [28], who found that the total (i.e., intravascular and parenchy-

Fig. 2. $\mathrm{CD} 3+$ extravascular $\mathrm{T}$ cells correlate with tau pathology in Alzheimer disease (AD) brains. A Representative micrographs illustrating tau (AT8 antibody) and A $\beta$ (6E10 antibody) pathology across brain regions of the same individual with AD. Examples of neuritic plaques (indicated by the arrowhead) and AT8-positive perikarya (arrows) are shown. Scale bars, $50 \mu \mathrm{m}$ (AT8) and $100 \mu \mathrm{m}$ (6E10). B Quantification of A $\beta$ (expressed as numbers of 6E10positive plaques) and tau (expressed as numbers of AT8-positive structures) in the hippocampi and mid frontal gyrus (MFG) of AD and control, nondemented samples. Tau pathology was significantly higher in $\mathrm{AD}$ samples compared to controls $(p<0.01$, 2 -way-ANOVA; $p<0.05$ in MFG, $p<0.001$ in hippocampi, Bonferroni's post hoc comparison). Hippocampi displayed a more se- mal) CD3+ T cells correlated with tau, but not $\mathrm{A} \beta$ 1-42, in the superior and middle temporal gyrus, MFG and inferior parietal lobule of nonimmunized $\mathrm{AD}$ patients. In addition, $\mathrm{T}$-cell invasion has been observed in frontotemporal dementia cases [20], strongly indicating a link between tau degeneration and T-cell invasion.

In contrast to what we observed in the $\mathrm{AD}$ brain samples, no correlation was found between T cells and tau pathology in nondemented controls, where instead a weak correlation existed with cerebral amyloid. It is important to note that although the nondemented control samples we analyzed displayed little to no tau pathology (as expected for early Braak stages), they harbored a high amyloid burden in both the hippocampus and the MFG. This finding was surprising, since the nondemented cases were rated as Consortium to Establish a Registry for Alzheimer's Disease (CERAD) A and B (corresponding to sparse and moderate frequencies of neuritic plaques, respectively). However, it should be noted that the hippocampus (where amyloid burden was almost identical between AD cases and controls) is not an area used for CERAD scoring. On the other hand, a nonsignificant trend towards a higher amyloidosis in $\mathrm{AD}$ cases was found in the MFG, as expected; the low number of subjects included in this study is likely the cause of missed statistical significance. Therefore, in this study we lacked a pure, pathology-free control group. However, our sample set might allow analysis of the effect of amyloid pathology alone (as seen in nondemented control subjects) versus amyloid-induced neurodegeneration. In this scenario, our data would indicate that cerebral amyloidosis is only weakly associated with T-cell infiltration (as seen in nondemented control samples); however, once neurodegeneration is in place, tau-dependent T-cell invasion will ensue. Further studies are warranted to test this hypothesis. vere tau pathology than MFG in AD samples (*** $p<0.001,2$-wayANOVA with Bonferroni's multiple comparisons). Amyloid pathology did not significantly differ across samples. C Correlation analysis of numbers of extravascular CD3+ T cells with $\mathrm{A} \beta$ (expressed as numbers of 6E10-positive plaques) and tau (expressed as numbers of AT8-positive structures) pathology in AD samples and nondemented controls, hippocampi, and MFG. The correlation is shown for all samples (hippocampi + MFG, AD and controls), as well as divided by diagnosis (rows) and brain region (columns). T-cell numbers significantly correlated with the number of AT8 immunoreactive structures in all of the samples $(p<0.001$, Pearson's correlation). Brain area- and diagnosis-specific effects were observed. ns, not significant. 


\section{Conclusions}

Low-grade $\mathrm{CD} 3+\mathrm{T}$-cell infiltration occurs in $\mathrm{AD}$ brains and is more pronounced in the hippocampi than in the MFG. Since the number of T cells in each sample correlated with tau, but not with amyloid pathology, we propose that $\mathrm{T}$-cell invasion belongs to the cascade of pathophysiologic events that follow $\mathrm{A} \beta$-induced neurodegeneration at early Braak stages, but become progressively $\mathrm{A} \beta$-independent in $\mathrm{AD}[29]$. Elucidating the interaction between neuronal degeneration and $\mathrm{T}$ cells might yield novel biomarkers for disease progression and targets for therapeutic intervention.

\section{Acknowledgments}

The authors would like to thank the NBB and the Institute of Pathology and Molecular Pathology of Zürich for providing valuable postmortem material. M.T.F. is the recipient of a research grant from Alzheimer's Research Switzerland. L.K. and T.K. are supported by the Velux Foundation (project No. 993).

\section{References}

1 Heneka MT, et al: Neuroinflammation in Alzheimer's disease. Lancet Neurol 2015;14: 388-405.

$\checkmark 2$ Town T, et al: T-cells in Alzheimer's disease. Neuromolecular Med 2005;7:255-264.

3 Lynch MA: The impact of neuroimmune changes on development of amyloid pathology: relevance to Alzheimer's disease. Immunology 2014;141:292-301.

4 Engelhardt B, et al: Vascular, glial, and lymphatic immune gateways of the central nervous system. Acta Neuropathol 2016;132: 317-338.

5 Wolf SA, et al: CD4-positive T lymphocytes provide a neuroimmunological link in the control of adult hippocampal neurogenesis. J Immunol 2009;182:3979-3984.

6 Ziv Y, et al: Immune cells contribute to the maintenance of neurogenesis and spatial learning abilities in adulthood. Nat Neurosci 2006;9:268-275.

7 Ousman SS, Kubes P: Immune surveillance in the central nervous system. Nat Neurosci 2012; 15:1096-1101.

-8 Itagaki S, McGeer PL, Akiyama H: Presence of T-cytotoxic suppressor and leucocyte common antigen positive cells in Alzheimer's disease brain tissue. Neurosci Lett 1988;91:259264.

-9 Rogers J, et al: Expression of immune systemassociated antigens by cells of the human central nervous system: relationship to the pathology of Alzheimer's disease. Neurobiol Aging 1988;9:339-349.

10 Togo $\mathrm{T}$, et al: Occurrence of $\mathrm{T}$ cells in the brain of Alzheimer's disease and other neurological diseases. J Neuroimmunol 2002;124: 83-92.
1 Parachikova A, et al: Inflammatory changes parallel the early stages of Alzheimer disease. Neurobiol Aging 2007;28:1821-1833.

$>12$ Grandjean J, et al: Complex interplay between brain function and structure during cerebral amyloidosis in APP transgenic mouse strains revealed by multi-parametric MRI comparison. Neuroimage 2016;134:1-11.

13 Braak H, et al: Stages of the pathologic process in Alzheimer disease: age categories from 1 to 100 years. J Neuropathol Exp Neurol 2011;70: 960-969.

14 Braak H, et al: Staging of Alzheimer diseaseassociated neurofibrillary pathology using paraffin sections and immunocytochemistry. Acta Neuropathol 2006;112:389-404.

15 Monsonego A, et al: Increased T cell reactivity to amyloid beta protein in older humans and patients with Alzheimer disease. J Clin Invest 2003; 112:415-422.

$>16$ Lueg G, et al: Clinical relevance of specific Tcell activation in the blood and cerebrospinal fluid of patients with mild Alzheimer's disease. Neurobiol Aging 2015;36:81-89.

17 Monson NL, et al: Elevated CNS inflammation in patients with preclinical Alzheimer's disease. J Cereb Blood Flow Metab 2014;34: 30-33.

18 Smolders J, et al: Characteristics of differentiated CD8(+) and CD4(+) T cells present in the human brain. Acta Neuropathol 2013; 126:525-535.

19 Ferretti MT, et al: T-cell brain infiltration and immature antigen-presenting cells in transgenic models of Alzheimer's disease-like cerebral amyloidosis. Brain Behav Immun 2016; 54:211-225.
20 Laurent C, et al: Hippocampal T cell infiltration promotes neuroinflammation and cognitive decline in a mouse model of tauopathy. Brain 2017;140:184-200.

21 Ferretti MT, et al: Intracellular Abeta-oligomers and early inflammation in a model of Alzheimer's disease. Neurobiol Aging 2012; 33:1329-1342.

22 Bellucci A, et al: Induction of inflammatory mediators and microglial activation in mice transgenic for mutant human P301S tau protein. Am J Pathol 2004;165:1643-1652.

23 Holman DW, Klein RS, Ransohoff RM: The blood-brain barrier, chemokines and multiple sclerosis. Biochim Biophys Acta 2011; 1812:220-230.

24 Grammas P, Ovase R: Inflammatory factors are elevated in brain microvessels in Alzheimer's disease. Neurobiol Aging 2001;22:837842 .

25 Merlini M, Wanner D, Nitsch RM: Tau pathology-dependent remodelling of cerebral arteries precedes Alzheimer's disease-related microvascular cerebral amyloid angiopathy. Acta Neuropathol 2016;131:737-752.

26 Liu CC, et al: Apolipoprotein E and Alzheimer disease: risk, mechanisms and therapy. Nat Rev Neurol 2013;9:106-118.

27 Shi Y, et al: ApoE4 markedly exacerbates taumediated neurodegeneration in a mouse model of tauopathy. Nature 2017;549:523527.

28 Zotova E, et al: Inflammatory components in human Alzheimer's disease and after active amyloid-beta42 immunization. Brain 2013; 136:2677-2696.

29 Jack CR Jr, et al: Tracking pathophysiological processes in Alzheimer's disease: an updated hypothetical model of dynamic biomarkers. Lancet Neurol 2013;12:207-216. 